\title{
UV PENETRATED CLUMPY MOLECULAR CLOUD CORES
}

\author{
JÜRGEN STUTZKI \\ I. Physikalisches Institut der Universität zu Köln, \\ Zülpicher Straße 77, D-5000 Köln 41, Fed. Rep. of Germany \\ REINHARD GENZEL, URS GRAF, ANDREW I. HARRIS, AMIEL STERNBERG \\ Max-Planck-Institut für Physik und Astrophysik, \\ Institut für extraterrestrische Physik, \\ D-8046 Garching b. München, Fed. Rep. of Germany \\ ROLF GÜSTEN \\ Max-Planck-Institut für Radioastronomie, \\ Auf dem Hügel 69, D-5300 Bonn 1, Fed. Rep. of Germany
}

\begin{abstract}
Recent observations of sub-mm and far-IR atomic fine structure and molecular rotational lines give evidence that due to the clumpiness of the molecular cloud cores the UV radiation from newly formed stars affects a very large fraction of the cloud material. Direct observations of the clumpy structure in M17 SW allow to derive several parameters of the clump distribution, in particular the clump mass spectrum and the volume filling factor. Implications of these results in regard to star formation are shortly discussed.
\end{abstract}

\section{UV Radiation and Molecular Clouds}

Far-UV radiation longward of the Lyman continuum edge (912 $\AA$ ) can escape from the HII region around newly formed stars and affect the surface of the parent molecular cloud. It dissociates most molecules, notably the most abundant ones, $\mathrm{H}_{2}$ and $\mathrm{CO}$, and ionizes several atomic species, in particular carbon $\left(\mathrm{C}^{+}\right)$. In addition the UV radiation directly heats the gas via two processes : first, fast photoelectrons ejected from dust grains collide with molecules, thus transferring the energy from the UV photons to the thermal energy of the gas. Second, and dominant at high densities, UV-pumped vibrational levels in $\mathrm{H}_{2}$ molecules collisionally deexcite to transfer their energy to the kinetic energy of the gas.

The UV-radiation thus creates a transition region, named photodissociation region (PDR), with a thickness of a few $A_{v}$ on the molecular cloud surface, where the chemical composition progressively changes from $\mathrm{HI}$ to $\mathrm{H}_{2}$, and in parallel, from $\mathrm{C}^{+}$to $\mathrm{C}^{0}$ to $\mathrm{CO}$. The temperature drops from about $1000 \mathrm{~K}$ at the cloud surface to the ambient cloud temperature of the UV shielded gas deep inside. The fine structure transitions of [CII] and [OI], as well as the mid- and high-J rotational transitions of $\mathrm{CO}$, all observable in the sub-mm and far-IR spectral range, are the dominant coolants of the gas.

Several groups have studied theoretically the structure of PDR's, starting with the early work by de Jong, Dalgarno and Boland (1980), through the extensive modelling of the Orion region by Tielens and Hollenbach $(1985 \mathrm{a}, \mathrm{b})$, and the more 
recent work by Sternberg and Dalgarno (1989), Burton et al. $(1988,1990)$ and Sternberg (1990). The intensities of the different fine structure transitions are generally reproduced rather nicely. However, all theoretical models fail to explain the large amount of warm molecular gas inferred from mid- and high-J CO and isotopic CO observations. This discrepancy is discussed further in the article by Graf et al. (this volume). Very recently Parmar, Lacy and Achtermann (priv. comm.) suceeded in the first observations of the $\mathrm{J}=4 \rightarrow 2(12 \mu \mathrm{m})$ and $\mathrm{J}=3 \rightarrow 1(17 \mu \mathrm{m})$ rotational transitions of molecular hydrogen in a strip map across the Orion Bar PDR. Their preliminary analysis gives hydrogen column densities and temperatures in apparently good agreement with the theoretical models discussed above.

\section{Clumpiness of Molecular Clouds and UV Radiation}

The different fine structure transitions of ionized carbon [CII] at $158 \mu \mathrm{m}$ and neutral atomic carbon [CI] at $370 \mu \mathrm{m}$ and $609 \mu \mathrm{m}$, and the mid- and high-J rotational transitions of $\mathrm{CO}$ (e.g. $\mathrm{J}=6 \rightarrow 5,7 \rightarrow 6,14 \rightarrow 13$ ) are ideally suited to study the $\mathrm{C}^{+} / \mathrm{C}^{0} / \mathrm{CO}$ transition structure of PDR's. They all show the emission regions of ionized and neutral atomic carbon, as well as warm molecular material traced by $\mathrm{CO}$, to be rather extended and to spatially coincide (Figure 1).

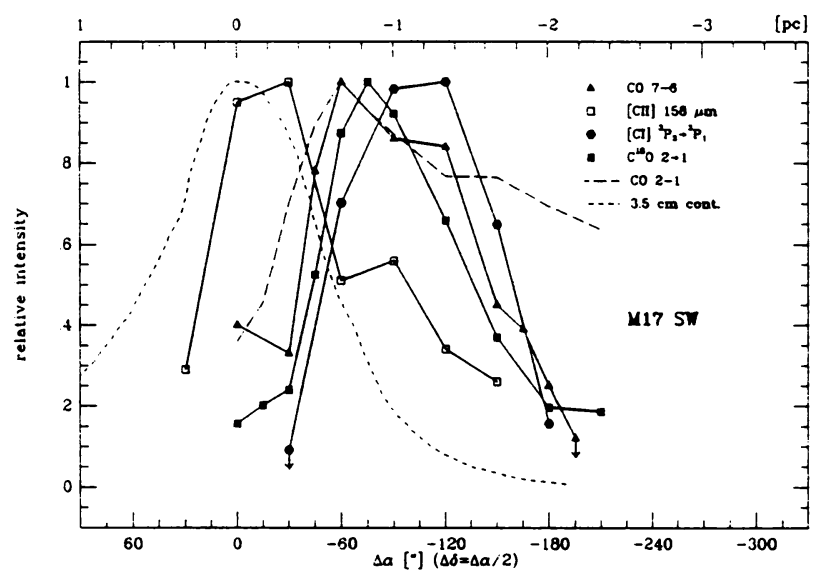

Fig. 1. Cut across the M17 SW HII region/molecular cloud interface showing the distribution of ionized, neutral atomic, and molecular carbon.

Moreover, the [CII] emission typically extends over regions where the average column density corresponds to $A_{v}$ of 10 to 100 in all regions observed, typical examples being Orion (Fig. 2), M17 SW (Fig. 3), the circumnuclear in the Galactic Center, W3 and NGC 1977. No UV photons able to ionize carbon can reach that far in, unless the cloud material is distributed very clumpy or filamentary, with a 


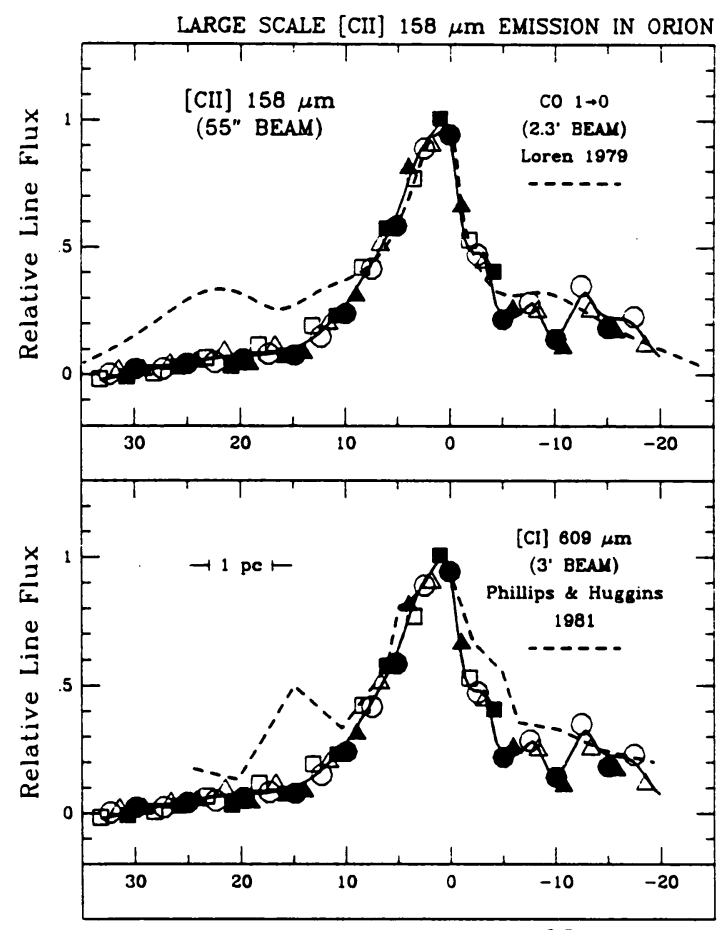

Right Ascension Offset [']

Fig. 2. Large scale distribution of $[\mathrm{CII}],[\mathrm{CI}]$ and $\mathrm{CO}$ emission in Orion (from Stutzki et al. 1990a). The different symbols denote the sets of strip scans with different chopper throw from which the $[\mathrm{CII}]$ data have been constructed.

high clump/interclump (column) density contrast. The observed intensity is then an average over many PDR's on the surfaces of individual, unresolved clumps.

This picture has several very attractive features. Detailed modeling, taking into account the attenuation of the UV-radiation with depth into the cloud by the blocking and scattering on clumps (Boissé 1990 presents a very detailed study of continuum radiative transfer in a clumpy medium), and incorporating the dependence of the emergent [CII] on the exciting UV intensity are very successful in reproducing not only the spatial distribution, but also the absolute intensity of the observed [CII] emission (Stutzki et al. 1988, Howe, Jaffe and Genzel 1990; Figure 4). The picture also naturally explains the coexistence of [CI] emission with the bulk of the molecular material (Keene et al. 1985, Genzel et al. 1988), without having to invoke special chemistry or untypically high initial carbon abundances.

Due to the clumpiness, the density in individual PDR's on clump surfaces is higher than estimated from the average density of the emission region. Higher densities help to explain the large amounts of warm molecular $\mathrm{CO}$ observed. At 


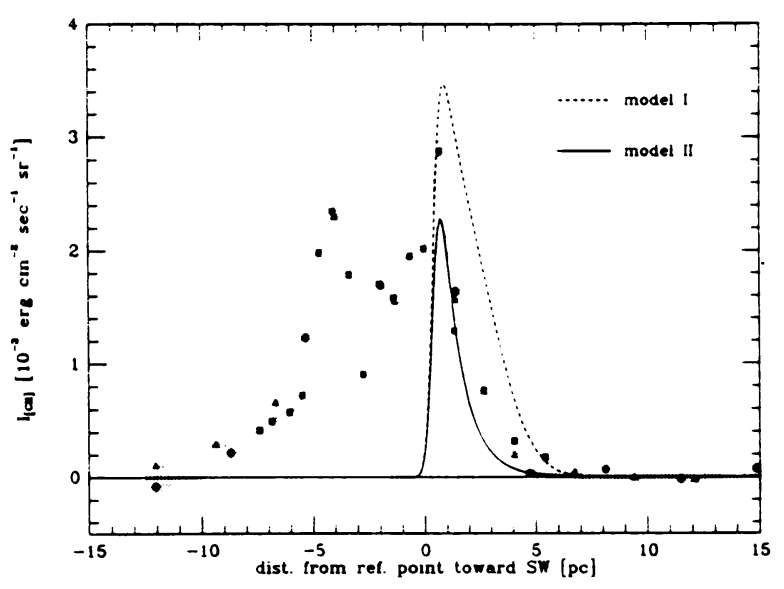

Fig. 3. The measured [CII] distribution compared with two simple models for the emission expected for a clumpy, UV penetrated cloud core (see Stutzki et al. 1988 for details).

higher densities, the molecular reformation rate is much faster and the molecular layer in the PDR is pushed out towards the higher temperature regime (Burton et al. 1990; Sternberg 1990). However, the extremely large column densities of warm molecular material implied by the recent ${ }^{13} \mathrm{CO} \mathrm{J}=6 \rightarrow 5$ detection (Graf et al. 1990, and this volume) still are not understood in the framework of theoretical PDR models.

The model of a clumpy, UV-penetrated cloud implies that the interpretation of $\mathrm{CO}$ lines in particular needs great care : the optical depth of CO rotational lines in the $\mathrm{mm}$ and sub-mm spectral regime is typically close to unity for column densities corresponding to $A_{v}$ of a few, that is for the thickness of individual PDR's. The temperature and the chemical composition of the emitting region thus varies strongly across the line formation region. Simple attempts to interpret the observed intensity ratios of more than two lines in a single component radiative transfer model (LVG or escape probablity codes) in consequence lead to intrinsically contradicting results (Dutrey et al. this volume). A more sophisticated approach, incorporating the temperature, density and chemical gradients on the clump surface (PDR) in the radiative transfer (Gierens, this volume), and comparing the observed intensity ratios with the approriate average over many clumps embedded in a (spatially varying) UV-field (Tauber and Goldsmith 1990) gives a straightforward explanation of the observed line intensities.

\section{Direct Observations of Clumpiness}

Several indirect, but nevertheless compelling arguments for the clumpy structure of molecular clouds are now commonly accepted. In some cases the observed bright- 
W3

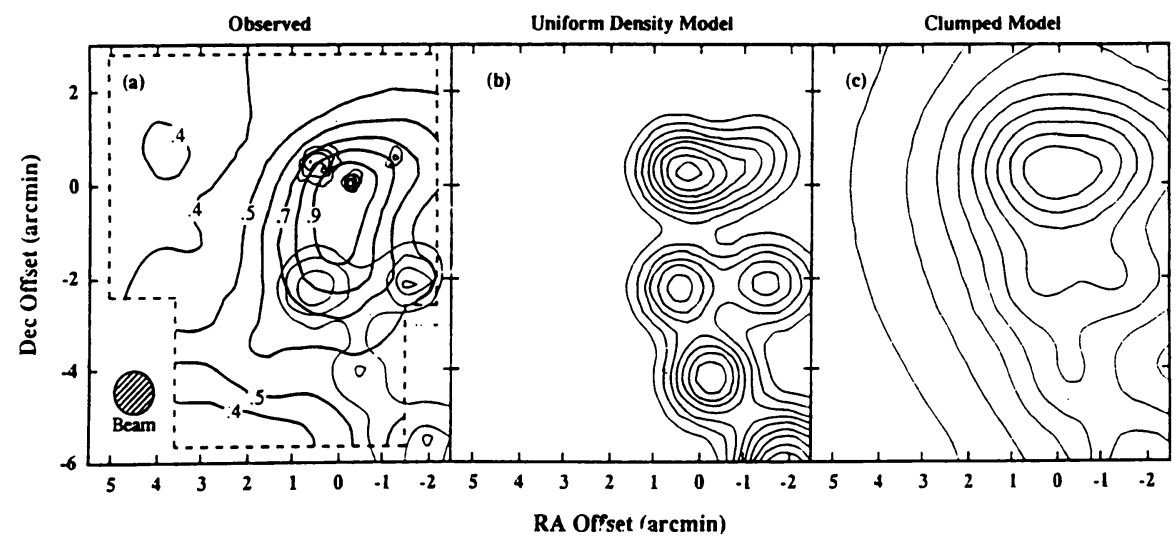

Fig. 4. The observed [CII] emission (left) of W3 compared with the one predicted assuming a homogeneous (mid), and a clumpy (right) cloud (from Howe, Jaffe and Genzel $1990)$.

ness temperature of a particular molecular transition is much lower than the excitation temperature independently derived from the comparison of other line ratios. Ammonia is a classical example (see Ho and Townes 1983). The missing intensity is then ascribed to a beam filling factor (typically around $20 \%$ ) of the emitting material, implying that the emitting material has structure on scales much smaller than the resolution of the observing telescope.

In other cases the density necessary for excitation of a particular line observed is much higher than the average density inferred from the ratio of column density and (assumed) source extent along the line of sight, again implying that the emission region is clumpy (this argument typically applies to multiline studies in CS, e.g. Snell et al. 1984, 1986, Evans et al. 1987, Mundy et al. 1986).

In an independent argument, the line profiles of different $\mathrm{CO}$ isotopes, in particular their width, which are observed to grow much less with optical depth than indicated by a simple curve of growth analysis, are successfully interpreted in a macroturbulent cloud model where the cloud consists of many individual, unresolved clumps of rather narrow intrinsic line width, but with a rather wider interclump velocity dispersion (Martin, Sanders and Hills 1984).

Only few direct observations of the clumpy structure of molecular clouds are published in the literature. Many of them refer to observations of very bright cen- 
tral regions, often in some molecular species for which it is not clear to what extent it traces the structure of the bulk molecular gas. $\mathrm{CO}$ observations or, due to the large optical depth of ${ }^{12} \mathrm{CO}$, rare isotopic $\mathrm{CO}$ observations with very high angular resolution should overcome this problem. Pérault, Falgarone and Puget (1985) published a study of nearby, cold clouds showing hierachical structure down to scales well below the Jeans length.

Stutzki and Güsten (1990) used the $\mathrm{C}^{18} \mathrm{O} J=2 \rightarrow 1$ transition at the. IRAM-30m telescope to map the molecular cloud core next to the edge on HII-region/molecular cloud interface M17 SW. The same region was simultaneously mapped in $\mathrm{C}^{34} \mathrm{~S}$ $\mathrm{J}=3 \rightarrow 2$ and $2 \rightarrow 1$ with somewhat poorer angular resolution. These data show small scale spatial and velocity structure, best seen in the comparison of two $0.5 \mathrm{~km} / \mathrm{s}$ wide velocity channel maps of the region (Figure 5). Clumps are identifiable down to the spatial resolution of the telescope (13" at $218 \mathrm{GHz}$ ). Individual clumps typically have line widths of 0.5 to $2 \mathrm{~km} / \mathrm{s}$, compared to an overall width of the position averaged line profile of $5 \mathrm{~km} / \mathrm{s}$. Note the very high peak brightness temperatures of even the rare isotopic $\mathrm{C}^{18} \mathrm{O} \mathrm{J}=2 \rightarrow 1$ emission, implying column densities of up to several times $10^{23} \mathrm{~cm}^{-2}$ in individual clumps.

Together with the typical clump dimensions of a few tenths of a pc, these high column densities immediately imply average densities in the $\mathrm{C}^{18} \mathrm{O}$ emission region of at least $10^{5} \mathrm{~cm}^{-3}$, much higher than the critical density necessary for excitation of the $\mathrm{J}=2 \rightarrow 1$ transition and rather similar to the densities inferred from CS multiline studies. The clump decomposition procedure discussed below confirms these high densities. The total amount of gas traced by $\mathrm{C}^{18} \mathrm{O}$ is similar to that traced by $\mathrm{C}^{34} \mathrm{~S}$. In addition, the $\mathrm{C}^{34} \mathrm{~S} \mathrm{~J}=3 \rightarrow 2$ and $\mathrm{C}^{18} \mathrm{O} \mathrm{J}=2 \rightarrow 1$ maps, once the latter is smoothed to the lower angular resolution of the former, look very similar. These results confirm that the bulk of the gas in M17 SW is in high density clumps.

Note that lower angular resolution observations in main isotopic $\mathrm{CO}$ lines can be very misleading under these circumstances. High angular resolution ${ }^{12} \mathrm{CO}$ spectra show very high peak brightness temperatures (around $100 \mathrm{~K}$ ) and deep absorption notches (Stutzki and Güsten 1990). Their shape varies rapidly from position to position, resulting in a rather smooth, almost Gaussian line profile when observed at an angular resolution of about 1'. The intensity observed with low angular resolution, typically $50 \mathrm{~K}$, which is usually interpreted as a measure for the gas kinetic temperature being in good agreement with the dust temperature, obviously has no direct relation to the global properties of the gas, but happens to result from a positional average over the much more complicated and intrinsically much brighter spectra described above.

With an appropriate clump decomposition procedure Stutzki and Güsten (1990) were able to separate the emission from M17 SW into about 170 individual clumps, assuming that the individual clumps have a Gaussian density and velocity distribution. The clump sizes and velocity widths closely follow the virial equilibrium relation. The volume filling factor is around 20 to $30 \%$, with slightly higher values towards the more central regions. 

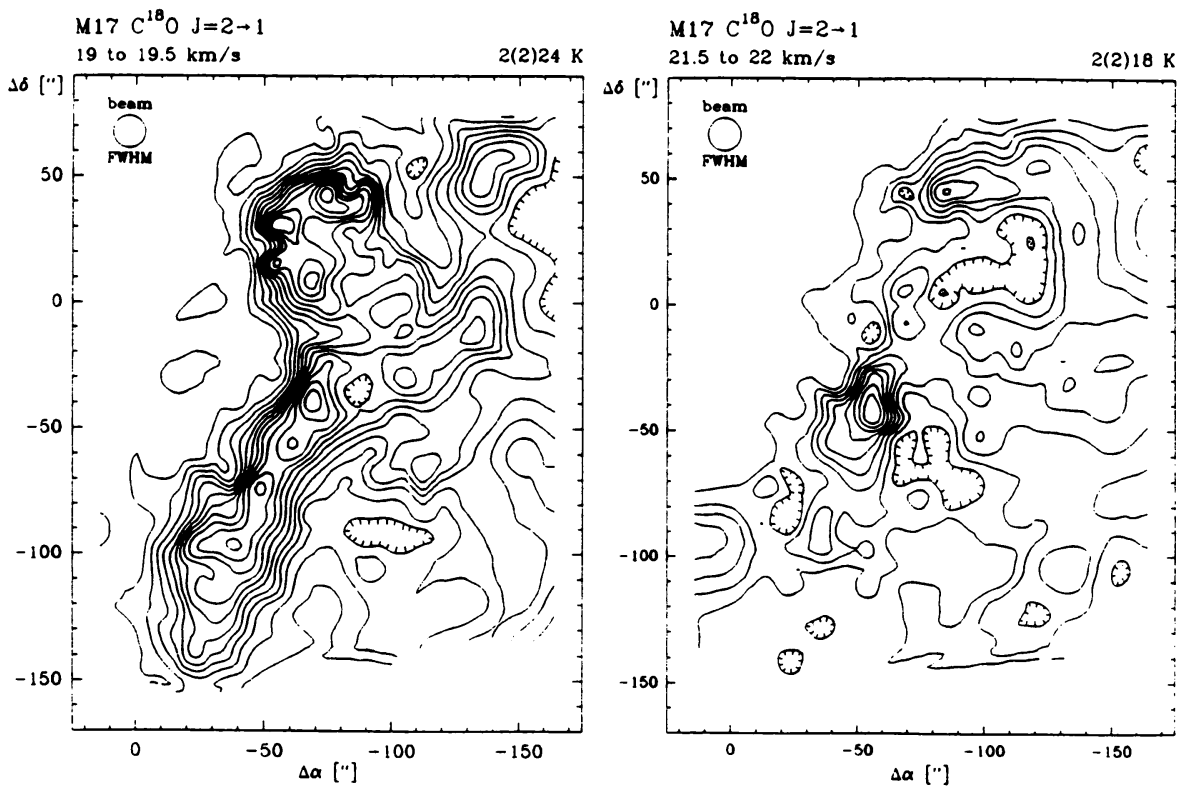

Fig. 5. Representative channel maps of the $\mathrm{M} 17 \mathrm{SW} \mathrm{C} \mathrm{C}^{18} \mathrm{O} \mathrm{J}=2 \rightarrow 1$ emission, delineating the clumpy nature of the cloud in the spatial and the velocity domaine. The contours are in steps of $2 \mathrm{~K}$, from 2 to $24 \mathrm{~K}$, in units of main-beam brightness temperature, averaged over $0.5 \mathrm{~km} / \mathrm{s}$ wide channels, centered at $v_{L S R}=19.25$ (left) and $21.75 \mathrm{~km} / \mathrm{s}$ (right).

This volume filling factor allows an estimate of the clump/interclump density contrast of $>23$, assuming that confusion with emission from lower density interclump gas starts at the $10 \%$ level of the peak emission. The extent of the observed [CII] emission, and hence the UV penetration depth into the cloud needs a density contrast of $>40$ and probably more like 100 .

The clumpiness of the molecular cloud structure does not seem to be related to the proximity of the M17 SW core to its exciting UV sources. A similar $\mathrm{C}^{18} \mathrm{O}$ map of a remote area of the M17 cloud, close to peak $\mathrm{C}$ in the notation of Elmegreen, Lada and Dickinson (1979), which is far off any detectable radio continuum emission or IR point source, shows the same clumpy structure (Stutzki et al. 1990b). Only the total (i.e. clump to clump) velocity dispersion is about a factor 2-3 lower, and hence the clump contrast is somewhat smeared out in individual velocity channel maps.

\section{Clump Mass Spectrum}

The derived clump masses show a spectrum $d N / d M \propto M^{-\alpha}$ with $\alpha=1.7$ over two 
orders of magnitude (Figure 6). A mass spectrum similar to the one found in the M17 SW study of Stutzi and Güsten (1990) is derived in many other cloud cores (Blitz 1987, Loren 1989, Lada 1990) and over a wide range of clump masses, from very large scale structures of neutral, atomic hydrogen clouds (Perry and Helfer 1972, Dickey and Garwood 1989), over giant molecular clouds (Casoli, Combes and Gérin 1984), down to fragments of order one solar mass and less. The derived values of the power law index $\alpha$ range between 1.1 and 1.8. A power law index of $\alpha=1.5$ is predicted in a simple coagulation and fragmentation scenario (Field and Saslaw 1965, Spitzer 1982, Nakano 1984) and depends only weakly on the details of the interclump interaction processes (Field and Hutchings 1968; Taff and Savedoff 1972, 1973; Arny and Weissman 1973; Silk and Takahashi 1979).

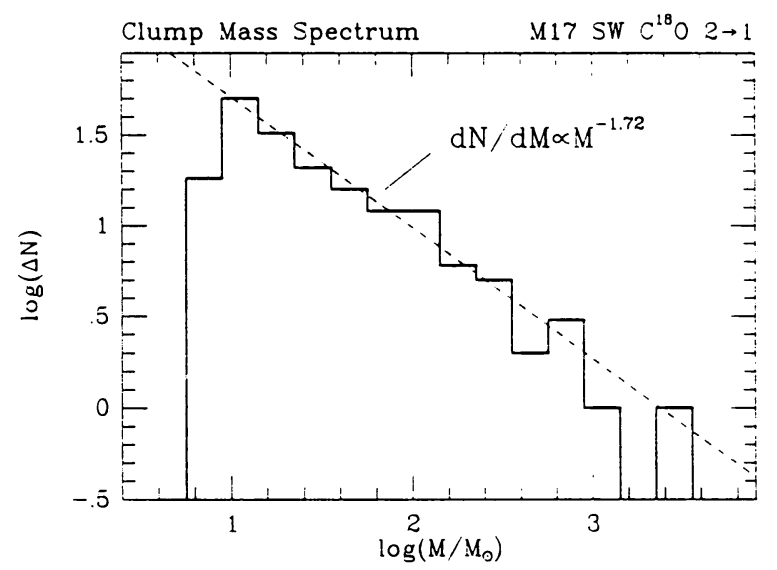

Fig. 6. The mass spectrum of the clumps identified in M17 SW is well fitted by a power law with an index $\alpha=1.7$.

Note that a power law with $\alpha=1.7$ together with an efficiency to convert clump mass into stellar mass $M_{*}=\epsilon M_{\text {clump }}^{p}$ translates into an initial mass function $d N / d M \propto M^{-q}$ where $q=(\alpha+p-1) / p$. For $\alpha=1.7$ as observed, and $p=0.5$, a plausible estimate based on present theoretical models of the protostellar evolution (Zinnecker 1989, and references therein), the resultant initial mass function has $q=2.4$, very close to the Salpeter initial mass function. One may thus speculate that the shape of the initial mass function is determined by the dynamical processes governing the fragmented structure of molecular clouds, combined with the efficiencies involved in forming individual stars, binaries and multiple systems with few members out of individual clumps. 


\section{Stability of the clumpy structure}

The picture emerging from the observational results discussed above shows that the molecular cloud core consists of individual, high density clumps with a volume filling factor of 20 to $30 \%$ and a very high density contrast between the clumps and the interclump medium. It is not clear at the moment what stabilizes the clumps against evaporating into the interclump phase. Notably, the pressure in the adjacent HII region, in the PDR envelopes and in the dense clump cores is quite comparable, about a few times $10^{6} \mathrm{~K} \mathrm{~cm}^{-3}$. If we postulate a neutral atomic interclump medium of comparable pressure to stabilize the clumps, the density contrast derived above predicts its density and temperature typically to be around $n=500-1000 \mathrm{~cm}^{-3}$ at $T=3000-6000 \mathrm{~K}$. Such a medium would be very difficult to detect with any presently known tool.

Alternatively, the clumps may all be marginally unstable against gravitational collapse and turn into a cluster of young stars within a few free fall times. This may eventually happen to all clumpy cores. The recent IR-camera observations (Lada 1990) showing dense stellar clusters around most OB groups observed, support the idea that the clumps discussed above eventually turn more or less at once into a cluster of young stars, and that this mode may be the dominant mode for star formation. The resultant picture is thus a more refined version of the old ideas of triggered star formation, where the first generation of a stellar cluster, due to the UV radiation from its brightest members, increases the pressure in the clumpy parent cloud (possibly by ablating more and more material from the clumps into the interclump medium), thus compresses the clumps until they become gravitationally unstable and turn into the next generation stellar cluster.

\section{Open Problems}

The present observations indicate that we have no satifactory understanding of the heating processes relevant in PDR's, missing some heating mechanism that is able to create rather large column densitites of warm molecular material. Also, in order for higher angular resolution observations to be useful, we need more versatile radiative transfer codes to study the radiation from a clumpy, turbulent medium.

The discussion above is largely based on observational data of only a few sources. Expansion of the observational data base, covering a larger variety of sources with different physical parameters, and equally important, observations with higher angular resolution, sufficient to resolve individual PDR surfaces on clumps, are necessary to understand the physical processes controlling the evolution of a clumpy molecular cloud core pentrated by UV radiation.

\section{References}

Arny, T., and Weissman, P., 1973, Astron. J. 78, 309. 
Blitz, L., 1987, in Physical Processes in Interstellar Clouds, eds. G.E. Morfill and M. Scholer, (Dordrecht : Reidel), p. 35.

Boissé, P., 1990, Astron. Astrophys. 228, 502.

Burton, M., Hollenbach, D., and Tielens, A., 1989, Proceedings of 22nd Eslab Symposium on Infrared Spectroscopy in Astronomy, Salamanca, 7-9 Dec. 1988 (ESA SP series).

Burton, M., Hollenbach, D., and Tielens, A., 1990, preprint.

Casoli, F., Combes, F., and Gérin, M., 1984, Astron. Astrophys. 113, 99.

de Jong, T., Dalgarno, A., and Boland, W., 1980, Astron. Astrophys. 91, 68.

Dickey, J.M., and Garwood, R.W., 1989, Astrophys. J. 341, 201.

Elmegreen, B.G., Lada, C.J., and Dickinson, D.F., 1979, Astrophys. J. 230, 415.

Evans, N.J., Mundy, L.G., Davis, J.H., and Vanden Bout, P., 1987, Astrophys. J. 312 , 344.

Field, G.B., and Hutchins, J., 1968, Astrophys. J. 153, 737.

Field, G.B., and Saslaw, W.C., 1965, Astrophys. J. 142, 568.

Genzel, R., Harris, A.I., Jaffe, D.T., and Stutzki, J., 1988, Astrophys. J. 332, 1043.

Graf, U.U., Genzel, R., Harris, A.I., Hills, R.E., Russell, A.P.G., and Stutzki, J., 1990, Astrophys. J. Letters, in press.

Ho, T.P.T., and Townes, C.H., 1985, Ann. Rev. Astron. Astrophys. 21, 239.

Howe, J., Jaffe, D.T., and Genzel, R., 1990, preprint.

Keene, J., Blake, G.A., Phillips, T.G., Huggins, P.J., and Beichman, C.A., 1985, Astrophys. J. 299, 967.

Lada, E., 1990, Ph.D. Thesis, University of Texas at Austin.

Loren, R.B., 1989, Astrophys. J. 338, 902.

Martin, H.M., Sanders, D.B., and Hills, R.E., 1984, Monthly Notices Roy. Astron. Soc. 208, 35.

Mundy, L.G., Snell, R.L., Evans, N.J., Goldsmith, P.F., and Bally, J., 1986, Astrophys. J. 306, 670 .

Nakano, T., 1984, Fund. Phys. 9, 139.

Pérault, M., Falgarone, E., and Puget, J.L., 1985, Astron. Astrophys. 152, 371.

Perry, J.F., and Helfer, H.L., 1972, Astrophys. J. 174, 341.

Sanders, D.B., Scoville, N.Z., and Solomon, P.M., 1985, Astrophys. J. 289, 373.

Silk, J., and Takahashi, T., 1979, Astrophys. J. 229, 242.

Snell, R.L., Mundy, L.G., Goldsmith, P.F., Evans, N.J., and Erickson, N.R., 1984, Astrophys. J. 276, 625 .

Snell, R.L., Goldmith, P.F., Ulich, B.L., Lada, C.J., Martin, R., and Schulz, A., 1986, Astrophys. J. 304, 780.

Spitzer, L., 1982, Searching between the Stars (New Haven: Yale University Press), pp. 148-151.

Sternberg, A., and Dalgarno, A., 1989, Astrophys. J. 338, 197.

Sternberg, A., 1990, in prep.

Stutzki, J., Stacey, G.J., Genzel, R., Harris, A.I., Jaffe, D.T., and Lugten, J.B., 1988, Astrophys. J. 332, 279.

Stutzki, J. et al., 1990a, in Submillimetre Astronomy. eds. G.D.Watt and A.S.Webster (Dordrecht : Kluwer), p. 269.

Stutzki, J., et al. 1990b, in preparation.

Stutzki, J., and Güsten, R., 1990, Astrophys. J. 356, 513.

Taff, L.G., and Savedoff. M.P., 1972, Monthly Notices Roy. Astron. Soc. 160, 89.

Taff, L.G., and Savedoff. M.P., 1973, Monthly Notices Roy. Astron. Soc. 164, 357.

Tauber, J.A., and Goldsmith, P.F., 1990, Astrophys. J. Letters 356, L61.

Tielens, A., and Hollenbach, D., 1985a, Astrophys. J. 291, 722.

Tielens, A., and Hollenbach, D., 1985b, Astrophys. J. 291, 747.

Zinnecker, H., 1989, in Evolutionary Phenomena in Galaxies, ed. J. Beckman (Cambridge : Cambridge University Press), p.115. 